\title{
Gauge Unification via Stable Brane Recombination
}

\author{
Kang-Sin Choi and Jihn E. Kim \\ School of Physics and Center for Theoretical Physics, \\ Seoul National University, Seoul 151-747, Korea \\ E-mail: ugha@phya.snu.ac.kr, jekim@phyp.snu.ac.kr
}

\begin{abstract}
We study the transition between parallel and intersecting branes on a torus. Spontaneous symmetry breaking of nonabelian gauge symmetry is understood as brane separation, and a more general intermediate deformation is discussed. We argue that there exists supersymmetry preserving transition and we can always have parallel branes as a final state. The transition is interpreted due to dynamics of the F- and D-string junctions and their generalization to (F, D p ) bound states. The gauge group and coupling unification is achieved, also as a result of supersymmetry. From the tadpole cancelation condition, we naturally have intersecting brane models as broken phases of Type I theory with $S O(32)$ gauge group.
\end{abstract}

KEYWORDS: brane recombination, brane junction, GUT, intersecting brane. 


\section{Contents}

1. Introduction 1

2. Intersecting branes 2

$2.1 \quad$ Brane separation and symmetry breaking 2

2.2 Intersecting brane world 1

2.3 Tilted but parallel branes 6

2.4 Intersecting branes at angles 7

3. Supersymmetry preserving brane recombination 8

3.1 Brane junction 8

3.2 Higher dimensions 10

3.3 Charge conservation and selection rules 11

4. Toward top-down approach 13

4.1 Gauge coupling unification 13

4.2 Including orientifold

5. Conclusions 16

\section{Introduction}

Unification of gauge couplings is an appealing idea. In field theory, it is neatly realized in grand unified theories [1] and also arises naturally in compactification of heterotic string theories [2]. In many models gauge couplings are unified at the string scale [3] (although the hypercharge embedding in such models in general cannot give a desirable value for the weak mixing angle, $\sin ^{2} \theta_{W}^{0}=\frac{3}{8}$ [4]).

In this paper, we show that the gauge group and coupling unifications are still present in the intersecting brane models. In Type I/II strings, the open strings describe gauge symmetry and they end on D-branes. If $N$ branes are stacked parallel, we have a $U(N)$ gauge symmetry out of which the $S U(N)$ coupling is the unified one. Their matrix-valued brane positions $X^{m}$ are translated into vacuum expectation values of gauge field $A_{m}^{\prime}$

$$
X^{m}=2 \pi \alpha^{\prime} A_{m}^{\prime},
$$

in the $T$-dual space. Depending on the eigenvalues, we have spontaneous symmetry breaking of gauge group, which is also translated into brane separation. If we can 
make use of the above idea of brane separation, we can understand unification. Some considered such unification partially in the parallel stacks [5] and even obtained some relations between couplings [6].

If a stack of $N_{1}$ branes and a $N_{2}$ branes intersect at angle, then the reduced four dimensional $U\left(N_{1}\right)$ and $U\left(N_{2}\right)$ couplings are independent. They are thus different in general because of the branes' different wrappings of internal world volumes.

We will show, in Section 3, that transition between parallel and intersecting branes, while preserving supersymmetry, can be achieved by turning on electric flux. Roughly speaking, we consistently introduce a more general control on the set of parameters describing the brane separation as

$$
X^{m}=\operatorname{diag}\left(f_{1}(X), f_{2}(X), \ldots, f_{N}(X)\right),
$$

where $f_{i}(X)$ describes a fluctuation of branes in $X^{m}$ direction and have a general shape. Although a general setup breaks supersymmetry, we recover supersymmetry by turning on electric flux, whose source is nothing but the F-string. This has been understood as string junction [7, 8, 9, 10, 11] and its higher dimensional generalization. The key property is that any deformation does not change the supersymmetry condiion or the BPS condition and the process is reversible. We can obtain the final state as a stack of (coincident or non-coincident) parallel branes.

To understand such a setup, one notes the crucial interplay between F- and Dstrings. The supersymmetry or BPS condition is interpreted as the balance condition at the junction they form. Their descriptions are similar except that their tensions differ by the factor of string coupling. In the $T$-dual picture, F-strings and D-branes become sources for electric and magnetic fluxes, respectively. Therefore, not only the D-brane but also the F-string dynamics are translated and unified into those of gauge fields. We will see that these together affect the four dimensional Yang-Mills coupling constant.

In Section 4, when we consider the Ramond-Ramond ( $\mathrm{R}-\mathrm{R}$ ) tadpole cancelation condition, which is the consistency condition of open strings, we observe that some class of models is naturally converted into some T-dual version of Type I string theory with gauge group $S O(32)$. We can reinterpret this result as the intersecting brane models being obtainable by compactifying Type I string with $S O(32)$.

\section{Intersecting branes}

\subsection{Brane separation and symmetry breaking}

When there are $N$ sheets of coincident $\mathrm{D} p$-branes, we have $U(N)$ gauge fields [12] Described by the Chan-Paton factor, a charge is attached on either end of an open string. An open string ending on branes transforms as the adjoint representation $\mathbf{N}^{2}$ of $U(N)$, because the open string between different branes behaves like a charged 
boson. It is shown that the lowest lying degrees along the brane are gauge fields and a transverse fluctuation is described by a scalar field. We have $T$-duality relations between them and the Dirac-Born-Infeld (DBI) action

$$
S=-T_{p} \int d^{p+1} x \operatorname{Tr} e^{-\Phi} \sqrt{-\operatorname{det}\left(G_{\mu \nu}+B_{\mu \nu}+2 \pi \alpha^{\prime} F_{\mu \nu}\right)}
$$

takes account of them. Expanding to the quadratic order in $\alpha^{\prime} F_{\mu \nu}$, it reduces to $(p+1)$-dimensional Yang-Mills (YM) action,

$$
S_{p}=-\frac{T_{p}\left(2 \pi \alpha^{\prime}\right)^{2}}{4 g_{\mathrm{s}}} \int d^{p+1} x \operatorname{Tr} F_{\mu \nu} F^{\mu \nu}
$$

with a potential of transverse scalar degrees. Here $T_{p}$ and $g_{\mathrm{s}}$ are the tension and Type II string coupling fixed by the vacuum expectation value (VEV) of dilaton. Therefore, the YM coupling is

$$
g_{p+1}^{2}=\frac{1}{2} g_{\mathrm{s}} T_{p}^{-1}\left(2 \pi \alpha^{\prime}\right)^{-2} .
$$

Location of branes $X^{m}$, which is matrix valued, is translated into gauge field $A_{m}^{\prime}$ in the $T$-dual space

$$
X^{m}=2 \pi \alpha^{\prime} A_{m}^{\prime}
$$

where $A_{m}^{\prime}=\sum A_{m}^{\prime a} T^{a}$. We put prime to indicate that the gauge field is in the $T$ dual space. We will use Greek indices for the worldvolume directions and lower case Roman indices for the transverse directions to the branes. The constant field can be always diagonalized by a suitable gauge transformation to

$$
X^{m}=\operatorname{diag}\left(a_{1}, a_{2}, \ldots, a_{N}\right) .
$$

From geometry, one can investigate the group structure. It corresponds to separation of branes located at $X^{m}=a_{1}, a_{2}, \ldots, a_{N}$. At generic values of $a_{i} \mathrm{~s}, U(N)$ breaks down to $U(1)^{N}$. Some of eigenvalues may be coincident, whose number we call $N_{k}$ where their sum is $N=\sum N_{k}$, so that the branes form separated stacks. It results in breaking $U(N)$ down to $U\left(N_{1}\right) \times U\left(N_{2}\right) \times \cdots \times U\left(N_{k}\right)$. The $U(1)$ factor in $U\left(N_{i}\right)$ corresponds to the overall translational symmetry of each stack. The transverse directions to the brane are compactified on small radii; thus the components $A_{m}^{\prime}$, in turn $X^{m}$, are decoupled and become scalar fields. It is understood as a Higgs mechanism that the adjoint Higgs $X^{m}$ develops a VEV. The potential is proportional to $\operatorname{Tr}\left[X^{m}, X^{n}\right]^{2}$, which comes from the T-dual of $\left(\partial_{[m} A_{n]}\right)^{2}$, and diagonalized matrix (2.5) gives flat directions for any values of $a_{i}$, thus supersymmetry is preserved at all the vacua. $X^{m}$ is the order parameter of symmetry breaking, which will be generalized to a more complicated setup. 


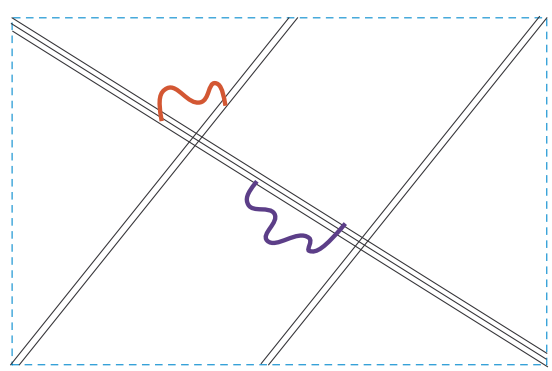

Figure 1: Intersecting branes with two families of $(\mathbf{3}, \mathbf{2})$ quarks charged under $S U(3) \times$ $S U(2)$.

\subsection{Intersecting brane world}

The above scalars form a real adjoint representation. To have a realistic model, we need chiral fermions. They naturally emerge from intersecting branes [13]. When we have $N_{a}$ and $N_{b}$ coincident branes intersecting with an angle, we obtain chiral fermions transforming as a bi-fundamental representation $\left(\mathbf{N}_{\mathbf{a}}, \mathbf{N}_{\mathbf{b}}\right)$ under $U\left(N_{a}\right) \times$ $U\left(N_{b}\right)$ (or $\left(\mathbf{N}_{\mathbf{a}}, \overline{\mathbf{N}}_{\mathbf{b}}\right)$, if instead we placed $N_{b}$ anti-branes). They are localized at the intersection, to minimize the energy proportional to the stretched string length.

In a typical setup [14, 15, 16] we have compact dimensions and each stack of branes wraps a cycle on it, and furthermore we have intersections between brane stacks in general. Fig. 11 shows a typical setup showing the basic features of intersecting branes. We have two and three slices of branes wrapping one-cycles in the two-torus, which account for $U(2)$ and $U(3)$ gauge groups, respectively. There are a finite number of intersection points in general. The same copy of intersections naturally explains the number of families and Yukawa couplings in the low energy theory are obtained geometrically [17, 18, 19]. The standard setup is a D6-brane wrapping the six-torus in Type IIA theory branes. Out of six, three internal dimensions wrap one-cycle in each two-torus and the remaining $(3+1)$ dimension takes account of our four dimensional world.

The typical intersecting brane world models suffer the following problems: the problem of gauge coupling unification, and the instability problem in non-supersymmetric models:

(i) The semisimple gauge group is obtained from the different stacks of branes in the construction, and hence the disconnected parts are not related to each other. Therefore, the gauge couplings are independent. Consider the dimensional reduction from the $(p+1)$ dimension to $(3+1)$ dimension

$$
-\frac{1}{4 g_{p+1}^{2}} \int d^{p+1} x\left|F_{\mu \nu}\right|^{2} \longrightarrow-\frac{V_{p-3}}{4 g_{p+1}^{2}} \int d^{4} x\left|F_{\mu \nu}\right|^{2}
$$



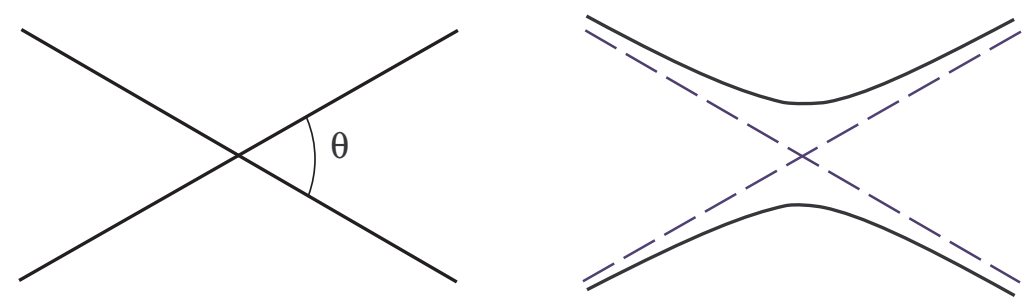

Figure 2: Without supersymmetry the intersecting branes are unstable. There is a recombined state that has a lower energy than that of the intersecting branes and decays to this lower energy state. The decay process goes on until the branes become flat.

Therefore, the four dimensional coupling constant squared is inversely proportional to the compact volume $V_{p-3}$ that each brane cycle wraps

$$
g_{4}^{2}=\frac{g_{p+1}^{2}}{V_{p-3}}
$$

For the coupling constant unification, there is a delicate issue on the nonstandard normalization of $U(1)$ couplings.

(ii) When we perform mode expansions of intersecting branes with suitable boundary conditions, we instantly see that the lowest excitation is the tachyon for a generic angle $\theta$ between branes,

$$
m^{2}=-\frac{\theta}{2 \pi \alpha^{\prime}}, \quad 0 \leq \theta \leq \pi .
$$

For more than one dimensions, $\theta=\sum( \pm 1) \theta_{i}$ is the sum of angles of the subtori $T^{2}$. This signals the brane instability [20], and the expansion is around the wrong vacuum. It is hoped that string field theory takes care of this instability problem. In this context, the tachyon condensation has been studied [21]. The supersymmetry route can be taken, where one can find some combinations of angles vanishing in higher dimensions for the lowest energy. Alternatively we might abandon supersymmetry and this tachyon phenomenon is used as a Higgs mechanism [22, in which case the electroweak scale $M_{Z}$ (or the Higgs mass) should be comparable with compactification scale $1 / R$ (or the separation of branes). However, from the point of view of unification and supersymmetry, this scenario is not so favorable. Another cure comes from turning on electric or magnetic field to recover supersymmetry, which is our viewpoint taken in the present paper.

We can understand the chirality of fermions as follows. Under the breaking $U(N) \rightarrow U\left(N_{a}\right) \times U\left(N_{b}\right)$, the branching rule for adjoint looks

$$
\mathbf{N}^{2} \rightarrow\left(\mathbf{N}_{\mathbf{a}}^{2}, \mathbf{1}\right)+\left(\mathbf{1}, \mathbf{N}_{\mathbf{b}}^{2}\right)+\left(\mathbf{N}_{\mathbf{a}}, \mathbf{N}_{\mathbf{b}}\right)+\left(\overline{\mathbf{N}}_{\mathbf{a}}, \overline{\mathbf{N}}_{\mathbf{b}}\right)
$$


where $N=N_{a}+N_{b}$. In the parallel brane case, the resulting massless fields are only the adjoints of the unbroken groups $\left(\mathbf{N}_{\mathbf{a}}^{\mathbf{2}}, \mathbf{1}\right)+\left(\mathbf{1}, \mathbf{N}_{\mathbf{b}}^{\mathbf{2}}\right)$. However, in the intersecting brane case, the would-be massive chiral fields $\left(\mathbf{N}_{\mathbf{a}}, \mathbf{N}_{\mathbf{b}}\right)$, sometimes called $X^{ \pm}, Y^{ \pm}$ bosons in the $S U(5)$ GUT for example, become massless at the intersecting point. Note that we should count the fermion $\left(\mathbf{N}_{\mathbf{a}}, \mathbf{N}_{\mathbf{b}}\right)$ only once because its complex conjugate representation corresponds to its CPT conjugate. This is the typical situation in string theory, because the complete state is formed with the right movers which provide the chirality [13, 23]. Note that the change of the metric (i.e. the complex structure) does not change the presence of chiral fermions and the spectrum, although it affects the nonvanishing masses of fermions. This reveals some structure that such setting can be embedded into the bigger group $U(N)$.

\subsection{Tilted but parallel branes}

Consider a rectangular two-torus $T^{2}$ with sides $L_{1}$ and $L_{2}$. We introduce the parametrization related to the winding number on the homology cycle $\left[\pi_{a}\right] \in H_{1}(T, \mathbf{Z})$. In two-torus we have two basis $[a]$ and $[b]$ each being an equivalence class of curves continuously shrinkable with the end points at each edges. We will use the winding vector $\left(n_{a}, m_{a}\right) \equiv n_{a}[a]+m_{a}[b]$ which at the same time denotes the $\mathrm{R}-\mathrm{R}$ charge components of the brane in the given torus. We restrict the discussion to the direct product of two-tori, so that every cycle is represented by the direct product of such vectors.

A single brane winding a cycle $(1, m)$ describes a $\mathrm{U}(1)$ gauge field. The consistency requires that the brane should be closed in the compact dimensions, not end. This tilted brane can be understood also as the $T$-dual of a two dimensional brane with a constant flux turned on 24, 25, 26]. It is noted that the DBI action is the unified description taking into account the both interpretations [27, 24]. In the $T$-dual space, we obtain $F_{21}=\frac{2 \pi}{L_{1} L_{2}} m$ where $m$ is quantized, $m \in \mathbf{Z}$. For the configuration of $U(1)$ bundle with the first Chern class $c_{1}=m$, it is nothing but the monopole or vortex quantization condition. Therefore, for such a tilted brane, there does not exist an extra modulus describing the angle because the shape of torus and the quantization condition totally determines the angle.

When we have a winding $(n, m)$, it cannot be described by an Abelian gauge group even though it is a single D-brane. We are forced to introduce the subgroup of the nonabelian gauge group $U(n)=(U(1) \times S U(n)) / \mathbf{Z}_{n}$ from two requirements: the shape of torus and the quantization condition of the angle $\theta$. The consistency

condition requires the quantized flux $F_{21}=\frac{2 \pi}{L_{1} L_{2}} \frac{m}{n}$ with $n, m \in \mathbf{Z}$, which has the same first Chern class $c_{1}=m$. We can always choose the gauge [28]

$$
\frac{1}{2 \pi \alpha^{\prime}} X^{2}=F_{21} X^{1}+\frac{2 \pi}{L_{2}} \operatorname{diag}\left(0, \frac{1}{n}, \ldots, \frac{n-1}{n}\right),
$$


up to a constant offsetting the origin. We have parallel branes with slope $\tan \theta=F_{12}$ from the $X^{1}$ axis and equal spacing $1 / n$, which corresponds to $U(1)^{n}$ obtained from spontaneous symmetry breaking of $U(n)$. We have parallel branes with slope

$$
\tan \theta=\frac{m}{n}
$$

Of course each has the same gauge coupling because winding volume in (2.7) is the same.

\subsection{Intersecting branes at angles}

Even when branes are intersecting with an angle, we have a similar order parameter description as done in (2.10). It is more convenient to describe in the $T$-dual space, using the dual gauge field $A_{\mu}$. Consider two sheets of D2-branes extended in the $X^{1}-X^{2}$ direction and the constant magnetic flux $F_{12}$ turned on it, which describes a $U(2)$ gauge theory. By an appropriate gauge transformation, we can always make the $F_{12}$ matrix diagonal. We may always choose a gauge $A_{1}=0$ and

$$
A_{2}=X^{1} F_{12}=\frac{2 \pi}{L_{1} L_{2}}\left(\begin{array}{cc}
p X^{1} & 0 \\
0 & -p X^{1}
\end{array}\right) \text {. }
$$

$T$-dualizing along the $X^{2}$ direction, we obtain

$$
X^{\prime 2}=2 \pi \alpha^{\prime} A_{2}=\frac{4 \pi^{2} \alpha^{\prime}}{L_{1} L_{2}}\left(\begin{array}{cc}
p X^{1} & 0 \\
0 & -p X^{1}
\end{array}\right)
$$

which is linear in $X^{1}$. Seen componentwise, this indicates that we have two pieces of D1-branes, having angles

$$
\theta= \pm \tan ^{-1} p
$$

relative to the $X^{1}$ axis. Here the magnetic flux $p$ is integrally quantized from the consistency condition and it is interpreted as the winding number in the dual picture.

We can generalize this idea to the case with more branes and more complicated magnetic fluxes. For a larger gauge group $U(N)$ broken to $U\left(N_{1}\right) \times U\left(N_{2}\right) \times \cdots \times$ $U\left(N_{k}\right)$, we can always diagonalze and choose gauge such that $A_{2}=X^{1} F_{12}$, as in $(2.12)$

$$
A_{2}=\frac{1}{2 \pi \alpha^{\prime}} X^{\prime 2}=\frac{X^{1}}{2 \pi \alpha^{\prime}} \operatorname{diag}\left(v_{1}, v_{2}, \ldots, v_{k}\right)
$$

up to constant offset. Corresponding to $U\left(N_{i}\right)$ for $i=1,2, \cdots$, we have $N_{i}$ identical value of $v_{i}$. Eventually it is equivalent to turning on a constant flux of $A_{2}$, which is Hermitian. After removing the diagonal constant corresponding the overall $U(1)$, we can always diagonalize the traceless $A_{2}$ by a unitary transformation. $v_{i}=m_{i} / N_{i}$ is quantized with the first Chern class $m_{i}$.

Ref. [9] considered the possibility of turning on constant off-diagonal components

of gauge fields. When we diagonalize $X$, the branes in this frame are in a form of 
reconnected ones, looking like Fig. 2. This is a special case arising from the fact that $X \mathrm{~s}$ are not mere numbers but matrices so that they are not commutative: the geometry is not clear but fuzzy. The off-diagonal components, which are proportional to some generators in another frame, are turned on by continuous Wilson lines. Because of the $X^{1}$ dependent terms in (2.13), the constant terms cannot be simultaneously diagonalized. The constant components do not change the BPS condition; thus the resulting theory is supersymmetric.

\section{Supersymmetry preserving brane recombination}

\subsection{Brane junction}

The supersymmetry transformation for gaugino is

$$
\delta \chi=\Gamma^{\mu \nu} F_{\mu \nu} \epsilon .
$$

Vanishing $\delta \chi$ corresponds to the BPS condition of Yang-Mills theory and the number of surviving supersymmetries is determined by that of the invariant spinor components.

Firstly, let us consider the simplest case of two dimensions along the $X^{1}-X^{2}$ directions, where two D1-branes (or D-strings) intersect at an angle. As seen before, in the $T$-dual picture, the tilted brane is responsible for the field strength $2 \pi \alpha^{\prime} F_{12}=$ $\partial_{1} X^{2}$ and the winding numbers are quantized. The branes being not parallel, there remains no supersymmetry, because there is no common solution to

$$
\Gamma^{12} F_{12} \epsilon=0
$$

for two different $F_{12}$ s. Nevertheless, we can reintroduce supersymmetry by turning on an electric flux [10, 11]

$$
X^{2}\left(X^{1}\right)=A_{0}\left(X^{1}\right) .
$$

Then, there is a solution of vanishing (3.1),

$$
\left(\Gamma^{12} F_{12}+\Gamma^{10} F_{10}\right) \epsilon=\left(\Gamma^{12}+\Gamma^{10}\right) F_{12} \epsilon=0
$$

or

$$
\left(\Gamma^{2}+\Gamma^{0}\right) \epsilon=0,
$$

which has a nontrivial solution. Supersymmetry is recovered by the component $F_{10}$ by compensating the back reaction of D-string deformation (3.3). This source for electric flux is interpreted as fundamental string (F-string), which has the desired tension [10]. Then the angle of the F-string about the D-string is given by

$$
\tan \theta=\frac{p g_{\mathrm{s}}}{q} .
$$



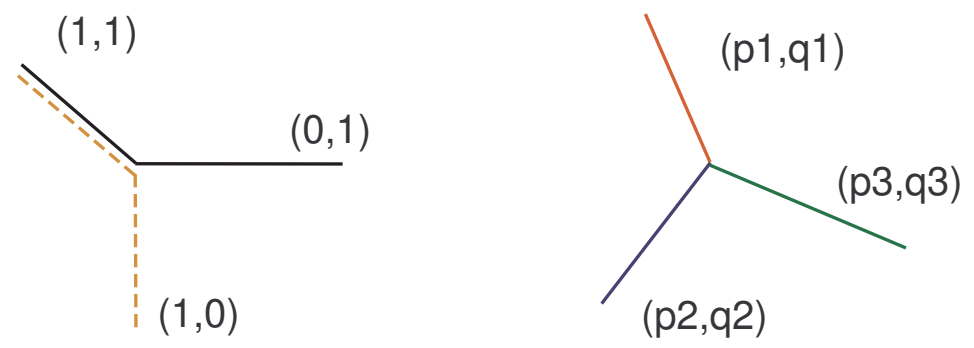

Figure 3: String junction. Its arbitrary deformation preserves the supersymmetry as long as the junction condition is satisfied. This is also understood as a (F, D p) bound state in higher dimensions.

Notice that this expression contains string coupling constant $g_{\mathrm{s}}$, thus is different from the setup with D-branes only (2.11). This is the condition of F1-D1 string bound state (Eq. (13.6.3) of [27)

$$
T_{p, q}=\sqrt{\left(p T_{1,0}\right)^{2}+\left(q T_{0,1}\right)^{2}}
$$

obtained from the supersymmetry algebra ((13.2.9) of [27]). This is rewritten as

$$
T_{p, q} \sin \theta=p T_{1,0}, \quad T_{p, q} \cos \theta=q T_{0,1}=\frac{q}{g_{\mathrm{s}}} T_{1,0},
$$

to reproduce (3.6). From this, we observe that the slope and the $\mathrm{F}$ and $\mathrm{D}$ charges are related as in Eqs. (3.6) and (3.8). This is nothing but the balance condition between the incoming and the outgoing branes, since at every point

$$
\sum p_{i}=0, \quad \sum q_{i}=0
$$

It is also called the string junction condition shown in Fig. 3. As long as this condition is satisfied, every string junction is a BPS state preserving some supersymmetries allowed by (3.5). It is natural to think of $p$ units of F-strings and $q$ units of Dstrings. When we compactify the spatial dimensions on $T^{2}$, we require the charge quantization condition. As seen in previous sections, we require $p$ and $q$ integers. We do not identify $(p, q)$ as the winding number $(n, m)$ but the identification is possible when we consider some small box encompassed or totally filled by a linear piece of the string.

In the balance condition between F- and D-strings, the Type II string coupling $g_{\mathrm{s}}$ enters because their tensions differ by the factor of $g_{\mathrm{s}}$. Comparing Eqs. (2.11) and (3.6), to have the F-D bound state we require $g_{\mathrm{s}}=1$. This does not ruin perturbertivity because coupling constant is also inversely proportional to volume, as in (2.7), which can vary. For the higher dimensional states where the BPS condition 

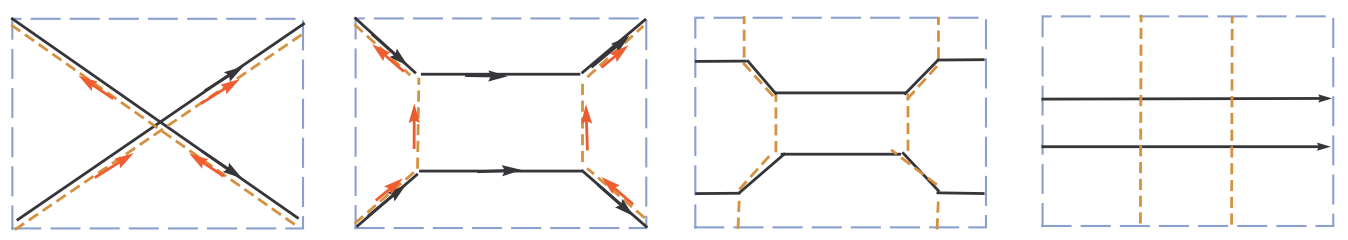

Figure 4: Transition of intersecting branes. Thick lines denote D-branes and dotted lines denote electric fluxes. Brane and charge orientations are denoted by arrows. Every step in this figure is on the same flat potential, therefore irreversible.

is saturated only by D-branes, we do not have such a $g_{\mathrm{s}}$ dependence. In fact, this system is converted into a bound state of D0-D2 with the same BPS condition (3.5), by $U$-duality transformations and $g_{\mathrm{s}}=1$ corresponds to the self-dual coupling.

The preserved supersymmetry is identical when we have F-strings along $X^{1}$,

$$
\epsilon=-\Gamma^{0} \Gamma^{2} \epsilon
$$

which is identical to (3.5). In addition, we have the D-brane along $X^{2}$ then

$$
\tilde{\epsilon}=\Gamma^{0} \Gamma^{1} \epsilon
$$

where $\tilde{\epsilon}$ is another supersymmetry parameter of the opposite chirality to $\epsilon$. This breaks additional half of the supersymmetries, generated by linear combination of left and right chiral supercharges $\epsilon Q+\tilde{\epsilon} \tilde{Q}$. The preserved supersymmetries are $1 / 4$ of the original supersymmetries and the same solution of (3.1) is preserved no matter how the intersection is deformed.

In Fig. 1 we show a simple but a typical situation for deformation of the intersecting branes. At every state the same supersymmetry is preserved and eventually the intersecting branes are always deformed into parallel branes without costing energy. In the last figure, we have parallel branes (with F-string winding). The studies in Refs. 32 have shown that various intermediate state corresponds to a distinct phase due to interplay between monopoles and instantons.

When we consider Chern-Simons couplings as well [11], we have a more general bending. We have an object that does not ruin supersymmetry condition (3.5), the $\mathrm{D}(-1)$ brane, charged under $\mathrm{R}-\mathrm{R}$ scalar. We can see the deformation as distribution of melted F-strings or electric field in the D-strings [9].

\subsection{Higher dimensions}

The above $(2+1)$ dimensional example is understood as a dimensional reduction of $(d+1)$ dimensional one. At first glance, this is not possible because there seems no $(d+1)$ dimensional objects carrying F-string (Neveu-Schwarz-Neveu-Schwarz (NSNS)) charge (except NS5 brane). However there have been arguments [30, 31] that 
continuous F-string filling in higher dimension can do such a job, called (F, D p) bound state. The direct argument goes as follows. Consider the above F1-D1 bound state. When we compactify one transverse direction and take its T-dual, we obtain F1-D2 bound state, where F1 string is melt to D2 brane. The fact of the 'boundedness' state is not affected in the $T$-dual picture as in the $\mathrm{D} p$ - $\mathrm{D} p^{\prime}$ bound state and also it can be verified by checking the number of supersymmetries. The shape of smaller brane is fuzzy and totally melt and the dimension of F-string is not important. Using $T$-duality iteratively in the transverse directions, we easily obtain an F1-D $p$ bound state. It has been argued in Ref. [30] and an explicit supergravity solution is obtained in Ref. [31] so that a supersymmetry condition similar to (3.7) is applied for such extended objects,

$$
T_{p,(m, n)}=T_{p} \sqrt{m^{2}+\frac{n^{2}}{g_{\mathrm{s}}^{2}}}
$$

where $T_{p}=\left(4 \pi^{2} \alpha^{\prime}\right)^{(11-p) / 2}$ is the pure $\mathrm{D} p$ brane tension [27]. Being the extension of F-string, it continuously smears out the transverse direction with density $T_{p}$, filling $(p-1)+1$ dimensional plane. Although it fills higher dimensional plane, it still remains as an F1 string and this fact evades the contradiction due to the fact that there is no higher rank NS-NS field other than $B_{\mu \nu}$. We can check the brane junction conditions (3.6, 3.8), in particular relations between $p$ and $q$ are the same. We have a direct dimensional reduction. This solution is valid only for bound states, which is different from the NS5-D5 nonbound states, for example.

Can we consider a more general solution, where the situation cannot be projected into $(1+2)$ dimensions? This corresponds to a typical intersecting brane model, where branes are wrapping 3-cycles in six torus $T^{6}$. We have an affirmative answer because in each two-subtorus we can always satisfy the junction conditions (3.6. 3.8) for arbitrary deformed D-branes, because the supersymmetry condition (3.5) holds regardless of the deformation of D-branes. It is also noted that the BPS-Laplace equation allows a linearly growing solution. In fact, in the case of noncompact dimensions, the linearly growing solution is a good and normalizable one [32].

\subsection{Charge conservation and selection rules}

It is important to note that $R-R$ and $N S-N S$ charges must be conserved, unless a D-brane is created nor annihilated. Any brane recombination takes place as long as each winding number is conserved

$$
\sum N_{a}\left(n_{a}, m_{a}\right)=\text { conserved. }
$$

Fig. 5 shows the typical features, where the sum of the charges in one direction vanishes, for example $(n, m)=(2,0)$. After the brane separation, the two slices of branes can describe either $U(2)$ or $U(1) \times U(1)$ depending on the separation, controlled by the (matrix-valued) VEV of modulus $X=2 \pi \alpha^{\prime} A$. This gives the selection 

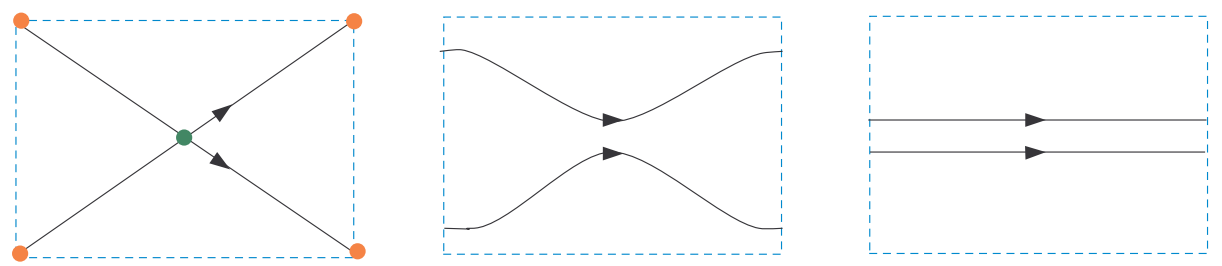

Figure 5: Recombination of branes on the torus. The transition $U(2) \leftrightarrow U(1) \times U(1)$ occurrs. The parallel case can be either $U(2)$ or $U(1) \times U(1)$ depending on the separation. Dots with the identical color in the initial state denote the same intersecting point. Arrows indicate the orientation of branes, or the Ramond-Ramond charges. The process is reversible because the geometry is determined by the D-flat moduli.
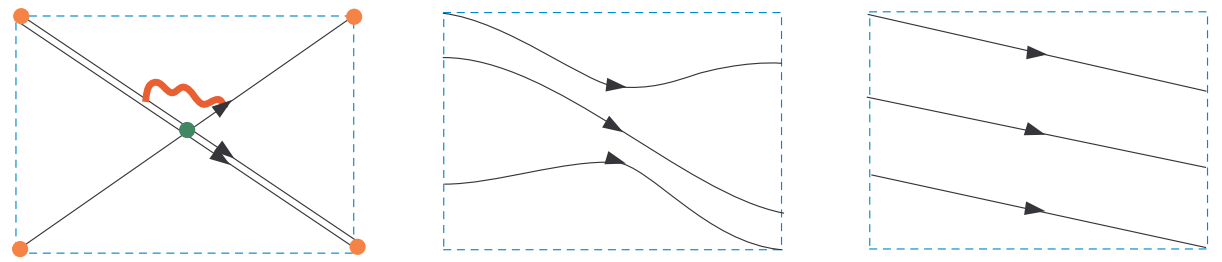

Figure 6: A more nontrivial setup compared to the preceeding figure. The intersecting case has the $U(2) \times U(1)$ gauge fields and a chiral fermion $(\mathbf{2}, \mathbf{1})$ localized at the intersection. We cannot make them coincident because the winding number along the vertical direction is preserved. With more branes forming stacks, we can explain the number of families. Alternatively, in the presence of orientifold plane, forced by tadpole cancelation, the winding number is summed up to zero corresponding to the branes coincident into one stack.

rules for the brane recombination process. We can freely move branes, guaranteed by the BPS saturated supersymmetry, in the way that the $R-R$ charge is conserved. This is also interpreted as the conservation of the brane winding number. We know that, when the strings merge or separate, the winding numbers are conserved in each direction of the torus.

This feature is shown in Figs. 5 and 6. Because we make a torus identifying sides of a rectangle, we have another intersection point on the edge. In Fig. 5, the $\mathrm{R}-\mathrm{R}$ charge or the winding numbers of the initial state is $(1,-1)$ and $(1,1)$ and that of the final state is $(2,0)$. The sum is preserved. The final state is parallel and is made coincident. The moduli space point corresponding to this coincident point allows the gauge enhancement to $U(2)$. A nontrivial case is the example shown in Fig. 6 where the sum relation is $2(1,-1)+(1,1)=(3,-1)$. In this case the three branes cannot move into a coincident point to one stack, because the winding number in the vertical direction is preserved with $n=-1$. A $U(3)$ enhancement does not occur. However, one can use the fact that the Lagrangian describing this situation is still a 
broken phase of $U(3)$.

\section{Toward top-down approach}

\subsection{Gauge coupling unification}

So far, we have discussed that there is a supersymmetry preserving deformation making all the branes parallel. Of course, the parallel and coincident brane states are described by the unique $U(n)$ gauge group and the gauge couplings are unified. (In the next subsection, we will consider the $\mathrm{R}-\mathrm{R}$ tadpole cancelation condition requiring negatively charged objects. When the typical solution of orientifold is introduced, we always end up with a situation with all the branes coincident.)

Note that the above vacua are supersymmetric and hence there needs no cost on deformation because they are in $D$-flat directions. It would be rather strange if the resulting four dimensional couplings, dependent on compact volume factor $V_{p}$ as in (2.7), differ between the parallel and the intersecting case (and intermediate steps). The puzzle arises because the naive expectation on volume dependence ignores the electric flux dependence which is not interpreted geometrically.

The desired form is read from the fluctuation spectrum analysis [28]. That is, we expand the gauge field $A_{\mu}$ around the intersecting brane background $A_{\mu}^{0}$,

$$
A_{\mu}=A_{\mu}^{0}+\delta A_{\mu} .
$$

Plugging it into the DBI action (2.1) and expanding up to the quadratic order in $\tilde{F}_{\mu \nu} \equiv \partial_{[\mu} \delta A_{\nu]}$, around

$$
K_{\mu}{ }^{\nu}=\left(1+2 \pi \alpha^{\prime} F^{0}\right)_{\mu}{ }^{\nu},
$$

we obtain

$$
\mathcal{L}=\sqrt{-\operatorname{det} K_{\mu \nu}}\left(K_{\mu \nu}^{-1} \tilde{F}^{\nu \lambda} K_{\lambda \sigma}^{-1} \tilde{F}^{\sigma \mu}+(\text { topological terms })\right) .
$$

Here $F_{\mu \nu}^{0}=\partial_{[\mu} A_{\nu]}^{0}$ from background branes and raising and lowering indices are done with the 'genuine' flat metric $\eta_{\mu \nu}$. The topological term does not contribute to the gauge coupling, and hence

$$
\mathcal{L}=\sqrt{-\operatorname{det} K_{\mu \nu}}\left(g^{\mu \nu} \tilde{F}_{\nu \lambda} g^{\lambda \sigma} \tilde{F}_{\sigma \mu}+\ldots\right) .
$$

Here the nonvanishing contribution of $K^{-1}$ is only the symmetric part [28], which we call 'metric' $g$. Note that the YM action cannot catch this volume dependence on $\sqrt{-\operatorname{det} K}$ and $g$ in (2.7) so the full DBI action is required.

When only the magnetic flux $f_{12}=f_{0}$ is turned on, we have

$$
K^{\mu \nu}=\left(\begin{array}{ccc}
-1 & 0 & 0 \\
0 & 1 & 2 \pi \alpha^{\prime} f_{0} \\
0 & -2 \pi \alpha^{\prime} f_{0} & 1
\end{array}\right) .
$$


(We deal with $(2+1)$ dimensional case and the generalization is straightforward.) Then we have

$$
g^{\mu \nu}=\operatorname{diag}\left(-1, \frac{1}{1+\left(2 \pi \alpha^{\prime} f_{0}\right)^{2}}, \frac{1}{1+\left(2 \pi \alpha^{\prime} f_{0}\right)^{2}}\right),
$$

which takes into account of the correct spacing of energy of spectrum when branes are tilted. We see that the overall factor becomes

$$
\sqrt{1+\left(2 \pi \alpha^{\prime} f_{0}\right)^{2}}=\sqrt{1+\tan ^{2} \theta}=\frac{1}{\cos \theta}
$$

where the second relation comes from the $T$-dual interpretation. Magnetic flux becomes geometry and gives rise to the extra factor $V_{p}=L_{1} L_{2} / \cos \theta$, which is nothing but the volume dependence of tilted branes from geometry, in (2.7). Note that the metric in the time direction is $g^{00}=-1$, thus it does not affect the canonical normalization of coupling.

When we have electric flux turned on in addition, satisfying the BPS condition $F_{01}=F_{12}=f_{0}$, we have

$$
K=\left(\begin{array}{ccc}
-1 & 2 \pi \alpha^{\prime} f_{0} & 0 \\
-2 \pi \alpha^{\prime} f_{0} & 1 & 2 \pi \alpha^{\prime} f_{0} \\
0 & -2 \pi \alpha^{\prime} f_{0} & 1
\end{array}\right)
$$

Remarkably the overall factor in (4.7), responsible for normalization of coupling, becomes

$$
\sqrt{-\operatorname{det} K}=1
$$

thus the volume dependence becomes to $V_{p}=1$, which is the same as the parallel brane case. Whatever shape the branes take, we have the same four dimensional gauge coupling; the gauge couplings are unified.

Of course, this modification is due to the effect of electric flux $F_{01}$. One may worry that there might be extra factor in $g^{00}$ which ruins the canonical normalization. However, when we plug in the new form of $K^{-1}$ and diagonalize it, the symmetric part in the 'metric' becomes

$$
g_{\text {diag }}^{\mu \nu}=\operatorname{diag}\left(-1, \frac{1}{1+2\left(2 \pi \alpha^{\prime} f_{0}\right)^{2}}, \frac{1}{1+2\left(2 \pi \alpha^{\prime} f_{0}\right)^{2}}\right) .
$$

confirming $g_{\text {diag }}^{00}=-1$. The nonabelian case shows essentially the same feature, although it cannot reproduce the correct infrared spectrum in the intersecting brane case.

\subsection{Including orientifold}

In open string theories, we need the Ramond-Ramond tadpole cancelation as a consistency condition for equation of motion [33]. The condition is derived from 

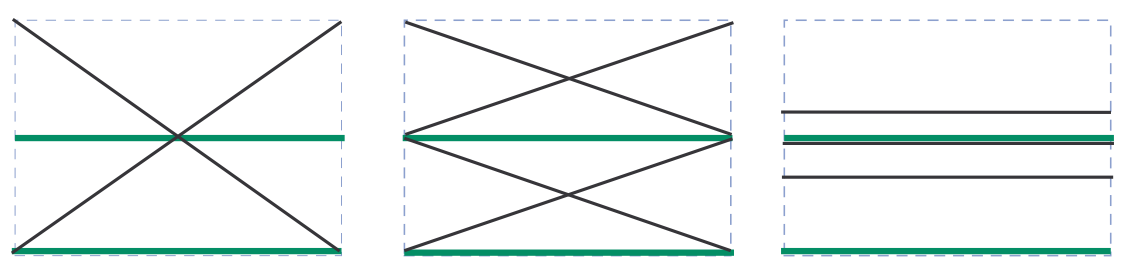

Figure 7: With orientifold the R-R tadpole cancelation condition is much more restricted. By the reflection symmetry, the sum of winding numbers transverse to the O-plane should vanish.

various 1-loop diagrams of open strings with Euler number one. Since it is dependent on the D-brane charge which winds topological cycles, it is converted to the charge conservation in the compact space. In our case of $(2+1)$ dimensional setup, the cancelation condition reads

$$
\left[\pi_{\text {total }}\right]=\sum N_{a}\left(m_{a}, n_{a}\right)=0
$$

For example, in Fig. 1, the winding number is $(1,1)$ for $U(3)$ stack and $(1,2)$ for $U(2)$ stack. We can verify that the sum of charge is not zero. To cancel the tadpole, we should insert a D-brane like object having the opposite $\mathrm{R}-\mathrm{R}$ charge. The best choice is the orientifold plane ( $\mathrm{O} p$ plane). Its tension is

$$
T_{\mathrm{O} p}=\mp 2^{p-5} T_{\mathrm{D} p}
$$

where the signs taking account of relative orientation with respect to D-branes. Therefore in effect we have a charged object of $\left(\mp 2^{p-5}, 0\right)$. Because of the reflection symmetry, the sum of $\mathrm{R}-\mathrm{R}$ charges is zero.

In addition, we should always have the 'mirror brane' with respect to the orientifold plane, as shown in Fig. 7. That is, for every $(n, m)$ winding state, we have an $(n,-m)$ winding state. Therefore, according to the selection rule we always have the state with a single stack of D-branes, aligned parallel to the direction of the orientifold plane. Since all are parallel, we can make them all coincident with orientifold planes. The final state is a gauge theory with $S O(2 n)$.

The dependence of $2^{p}$ is interpreted as follows. Because the orientifold is related by $\mathbf{Z}_{2}$ orbifold fixed points, we have twice the number of $\mathbf{Z}_{2}$ fixed points when we compactify a dimension. For $p=9$, for the space-filling orientifold the charge is 32 times that of the D-brane. This is nothing but the setup we obtain Type I theory out of Type IIB theory, by moding out the chirality. It strongly suggests that therefore many models are obtained from Type I theory with SO(32) gauge group.

Even if we include orientifold planes in various dimensions, the meaningful setup arises as T-dual of Type I string with $\mathrm{O} 5$ plane (also and/or O1 planes), because to 
preserve the number of supersymmetry we require the number of Neumann-Dirichlet direction (which is invariant quantity under $T$-dual) to be a multiple of four.

Of course, all the setups of intersecting branes at angles are not obtained in this way, because the final intersecting states considered here have more supersymmetries than $\mathcal{N}=1$. Nevertheless, we observe the following lesson learnt from the traditional compactification of heterotic strings. First, many supersymmetric models rely on the same supersymmetries, namely the small number of supersymmetries are obtained not by the unstable setup of intersection but the compactificaton on manifold with a certain holonomy. The realistic four supersymmetries $(\mathcal{N}=1$ in four dimensions $)$ is obtained by the orbifold projection [34 such as $T^{6} / \mathbf{Z}_{N}$ or $T^{6} / \mathbf{Z}_{M} \times \mathbf{Z}_{N}$ [35], but with the explicit supersymmetry breaking setup. Also, we have experienced that many vacua of heterotic string [23, 36] is connected when we consider $(2,2)$ compactification [37. We may conjecture that a vacuum of less supersymmetric setup is also connected to a more symmetric setup.

\section{Conclusions}

In this paper we studied a supersymmetry preserving deformation of branes. The symmetry of nonabelian gauge group is understood as parallel branes. Their separation gives rise to gauge symmetry breaking and can be interpreted as spontaneous symmetry breaking along flat directions. With more general deformations we introduce a deeper understanding on the symmetry breaking. We have the selection rule for charge conservation and showed the possible deformation to the state where all branes are parallel. It is described by a unique gauge group and suggests the unification of gauge coupling constants.

Supersymmetry is maintained by turning on a suitable electric flux, which is interpreted as a F-string bound state or string junction. We can generalize this idea to higher dimensions to form a (F, D p) bound state and junction. Since this process does not ruin supersymmetry, we naturally maintain the supersymmetry at the high energy scale when we consider this process. This is due to the interplay between electric and magnetic flux.

Remarkably, in this situation, we have shown that at every step, we have the same four dimensional gauge couplings, contrary to the conventional case when we have only magnetic flux. Thus we achieve gauge and coupling unification.

To cancel the Ramond-Ramond tadpole amplitude, we introduce orientifold planes. We have then the transition to the state where totally parallel and coincident branes along some coordinates and this is thought as some $T$-dual state of Type I string theory having gauge group $S O(32)$.

Here, we discussed only the cases for toroidal compactification and have not done the orbifold cases. However, we might conjecture that, like in the heterotic string theory, the realistic models seem to originate from a single theory. In this scenario, 
supersymmetry breaking is done by compactification on Calabi-Yau manifolds or orbifolds. In this sense, this paper provides a relevant scenario of top-down approach in the brane setup.

\section{Acknowledgments}

KSC is grateful to Daniel Cremades, Seungjoon Hyun, Hyung Do Kim, and Michele Trapletti for useful discussions and especially to Koji Hashimoto for motivation and answers to important questions. This work is supported in part by the KRF Sundo

Grant No. R02-2004-000-10149-0, the KRF ABRL Grant No. R14-2003-012-01001-0 and the BK21 program of Ministry of Education.

\section{References}

[1] H. Georgi, H. R. Quinn, and S. Weinberg, Hierarchy of interactions in unified gauge theories, Phys. Rev. Lett. 33 (1974) 451.

[2] Z. Kakushadze and S. H. H. Tye, Three family SO(10) grand unification in string theory, Phys. Rev. Lett. 77 (1996) 2612;

J. E. Kim, $Z_{3}$ orbifold construction of $S U(3)^{3} G U T$ with $\sin ^{2} \theta_{W}=\frac{3}{8}$, Phys. Lett. 564 (2003) 35 and 591 (2004) 119;

V. Braun, Y.-H. He, B. A. Ovrut, and T. Tantev, A heterotic standard model, Phys. Lett. 252B (2005) 252, hep-th/0501070.

[3] L. E. Ibanez, J. E. Kim, H. P. Nilles, and F. Quevedo, Orbifold compactifications with three families of $S U(3) \times S U(2) \times U(1)^{N}$, Phys. Lett. 191 (1987) 282;

J. A. Casas and C. Munoz, Three generation $S U(3) \times S U(2) \times U(1)^{N}$ models from orbifolds, Phys. Lett. 209 (1988) 214;

A. E. Faraggi, Aspects of nonrenormalizable terms in a superstring derived standard-like model, Nucl. Phys. B403 (1993) 101.

[4] R. Blumenhagen, M. Cvetic, P. Langacker, and G. Shiu, Toward realistic intersecting D-brane models, hep-th/0502005 (2005).

[5] M. Cvetic, P. Langacker, T. j. Li and T. Liu, D6-brane splitting on Type IIA orientifolds, Nucl. Phys. B 709, 241 (2005) [arXiv:hep-th/0407178].

[6] R. Blumenhagen, D. Lust and S. Stieberger, Gauge unification in supersymmetric intersecting brane worlds, JHEP 0307, 036 (2003) [arXiv:hep-th/0305146].

[7] J. H. Schwarz, An SL(2,Z) multiplet of Type IIB superstrings, Phys. Lett. B 360, 13 (1995) [Erratum-ibid. B 364, 252 (1995)] [arXiv:hep-th/9508143];

[8] E. Witten, Bound states of strings and p-branes, Nucl. Phys. B 460, 335 (1996) [arXiv:hep-th/9510135]. 
[9] K. Hashimoto and W. Taylor, Strings between branes, JHEP 0310, 040 (2003) [arXiv:hep-th/0307297].

[10] C. G. Callan and J. M. Maldacena, Brane death and dynamics from the Born-Infeld action, Nucl. Phys. B 513, 198 (1998) [arXiv:hep-th/9708147].

[11] K. Dasgupta and S. Mukhi, BPS nature of three string junctions, Phys. Lett. B 423, 261 (1998) [arXiv:hep-th/9711094].

[12] E. Witten, Solutions of four dimensional field theories via M-theory, Nucl. Phys. B 500, 3 (1997) [arXiv:hep-th/9703166];

J. Polchinski, Dirichlet branes and Ramond-Ramond charges, Phys. Rev. Lett. 75, 4724 (1995) [arXiv:hep-th/9510017].

[13] M. Berkooz, M. R. Douglas and R. G. Leigh, Branes intersecting at angles, Nucl. Phys. B 480, 265 (1996) [arXiv:hep-th/9606139].

[14] L. E. Ibanez, F. Marchesano and R. Rabadan, Getting just the standard model at intersecting branes, JHEP 0111, 002 (2001) [arXiv:hep-th/0105155];

G. Aldazabal, S. Franco, L. E. Ibanez, R. Rabadan and A. M. Uranga, Intersecting brane worlds, JHEP 0102, 047 (2001) [arXiv:hep-ph/0011132].

[15] A. M. Uranga, Chiral four dimensional string compactfications with intersecting D-branes, Class. Quant. Grav. 20, S373 (2003) [arXiv:hep-th/0301032].

[16] R. Blumenhagen, M. Cvetic, P. Langacker and G. Shiu, Toward realistic intersecting D-brane models, arXiv:hep-th/0502005.

[17] G. Aldazabal, S. Franco, L. E. Ibanez, R. Rabadan and A. M. Uranga, JHEP 0102 (2001) 047 [arXiv:hep-ph/0011132];

D. Cremades, L. E. Ibanez and F. Marchesano, Yukawa couplings in intersecting D-brane models, JHEP 0307, 038 (2003) [arXiv:hep-th/0302105].

[18] S. Hamidi and C. Vafa, Interactions on orbifolds, Nucl. Phys. B 279, 465 (1987).

[19] A. Font, L. E. Ibanez, H. P. Nilles and F. Quevedo, Degenerate orbifolds, Nucl. Phys. B 307, 109 (1988)

[20] K. Hashimoto and S. Nagaoka, Recombination of intersecting D-branes by local tachyon condensation, JHEP 0306, 034 (2003) [arXiv:hep-th/0303204];

J. Erdmenger, Z. Guralnik, R. Helling and I. Kirsch, A world-volume perspective on the recombination of intersecting branes, JHEP 0404, 064 (2004) [arXiv:hep-th/0309043].

[21] A. Sen, Tachyon condensation on the brane anti-brane system, JHEP 9808, 012 (1998) [arXiv:hep-th/9805170];

A. Sen, Universality of the tachyon potential, JHEP 9912, 027 (1999) [arXiv:hep-th/9911116]. 
[22] D. Cremades, L. E. Ibanez and F. Marchesano, Intersecting brane models of particle physics and the Higgs mechanism, JHEP 0207, 022 (2002) [arXiv:hep-th/0203160];

C. Kokorelis, Exact standard model structures from intersecting D5-branes, Nucl. Phys. B 677 (2004) 115 [arXiv:hep-th/0207234].

[23] K. S. Choi, Spectrum of heterotic string on orbifold, Nucl. Phys. B 708, 194 (2005) [arXiv:hep-th/0405195].

[24] C. Bachas, D-brane dynamics, Phys. Lett. B 374, 37 (1996) [arXiv:hep-th/9511043].

[25] R. Blumenhagen, L. Goerlich, B. Kors and D. Lust, Noncommutative compactifications of Type I strings on on tori with magnetic background flux, JHEP 0010, 006 (2000) [arXiv:hep-th/0007024].

[26] M. Bianchi and E. Trevigne, The open story of magnetic fluxes, arXiv:hep-th/0502147.

[27] J. Polchinski, String theory. Vol. 2: Superstring theory and beyond (Cambridge Univ. Press, 1998) 531 pp.

[28] A. Hashimoto and W. Taylor, Fluctuation spectra of tilted and intersecting D-branes from the Born-Infeld action, Nucl. Phys. B 503, 193 (1997) [arXiv:hep-th/9703217].

[29] R. Rabadan, Branes at angles, torons, stability and supersymmetry, Nucl. Phys. B 620, 152 (2002) [arXiv:hep-th/0107036];

P. van Baal, , Commun. Math. Phys. 85, 529 (1982).

[30] J. X. Lu and S. Roy, (m,n) string-like Dp-brane bound states, JHEP 9908, 002 (1999) [arXiv:hep-th/9904112].

[31] J. X. Lu and S. Roy, Nonthreshold (F, Dp) bound states, Nucl. Phys. B 560, 181 (1999) [arXiv:hep-th/9904129].

[32] O. Aharony, A. Hanany and B. Kol, , JHEP 9801, 002 (1998) [arXiv:hep-th/9710116];

A. Hanany and E. Witten, Type IIB superstrings, BPS monopoles, and three-dimensional gauge dynamics, Nucl. Phys. B 492, 152 (1997) [arXiv:hep-th/9611230];

O. Aharony and A. Hanany, Branes, superpotentials and superconformal fixed points, Nucl. Phys. B 504, 239 (1997) [arXiv:hep-th/9704170];

T. Kitao, K. Ohta and N. Ohta, Three-dimensional gauge dynamics from brane configurations with $(p, q)$-fivebrane, Nucl. Phys. B 539, 79 (1999) [arXiv:hep-th/9808111].

[33] R. Rabadan and F. Zamora, Dilaton tadpoles and D-brane intersections in compact spaces, JHEP 0212, 052 (2002) [arXiv:hep-th/0207178];

J. Polchinski and Y. Cai, Consistency of open superstring theories, Nucl. Phys. B 296, 91 (1988). 
[34] L. J. Dixon, J. A. Harvey, C. Vafa and E. Witten, Strings on orbifolds 2, Nucl. Phys. B 274, 285 (1986).

[35] See, for example,

M. Cvetic, G. Shiu and A. M. Uranga, Three family supersymmetric standard-like models from intersecting brane worlds, Phys. Rev. Lett. 87, 201801 (2001) [arXiv:hep-th/0107143];

S. Forste, G. Honecker and R. Schreyer, Orientifolds with branes at angles, JHEP 0106, 004 (2001) [arXiv:hep-th/0105208].

For more references, see Ref. [16].

[36] K. S. Choi, S. Groot Nibbelink and M. Trapletti, Heterotic SO(32) model building in four dimensions, JHEP 0412, 063 (2004) [arXiv:hep-th/0410232];

K. S. Choi, K. Hwang and J. E. Kim, Dynkin diagram strategy for orbifolding with Wilson lines, Nucl. Phys. B 662, 476 (2003) [arXiv:hep-th/0304243].

[37] L. E. Ibanez, Some Recent Results In (2,2) And (0,2) Heterotic String Compactifications, CERN-TH-5405/89, in in Proc. of 3rd Regional Conf. on Mathematical Physics, Islamabad, Pakistan (1989) and references therein. 